\title{
VARIOUS LIPSCHITZ-LIKE PROPERTIES FOR FUNCTIONS AND SETS I: DIRECTIONAL DERIVATIVE AND TANGENTIAL CHARACTERIZATIONS*
}

\author{
RAFAEL CORREA $^{\dagger}$, PEDRO GAJARDO $^{\ddagger}$, AND LIONEL THIBAULT ${ }^{\S}$
}

\begin{abstract}
In this work we introduce for extended real valued functions, defined on a Banach space $X$, the concept of $K$ directionally Lipschitzian behavior, where $K$ is a bounded subset of $X$. For different types of sets $K$ (e.g., zero, singleton, or compact), the $K$ directionally Lipschitzian behavior recovers well-known concepts in variational analysis (locally Lipschitzian, directionally Lipschitzian, or compactly epi-Lipschitzian properties, respectively). Characterizations of this notion are provided in terms of the lower Dini subderivatives. We also adapt the concept for sets and establish characterizations of the mentioned behavior in terms of the Bouligand tangent cones. The special case of convex functions and sets is also studied.
\end{abstract}

Key words. directional derivative, tangent cone, directionally Lipschitzian function, compactly epi-Lipschitzian function, epi-Lipschitzian set, compactly epi-Lipschitzian set, multidirectional mean value inequality

AMS subject classifications. Primary, 26A24, 49J52; Secondary, 49J52

DOI. $10.1137 / 080738271$

1. Introduction. Besides the usual concepts of locally Lipschitzian functions, various new Lipschitz-like properties for extended real valued functions and sets, introduced during the last 30 years, have played a fundamental role in recent developments of optimization and variational analysis. The first extension of the original Lipschitzian behavior property of a function is due to Rockafellar, who introduced in $[26,27,28]$ the concepts of directionally Lipschitzian functions and epi-Lipschitzian sets. A set $S$ of $\mathbb{R}^{n}$ is defined in [26] to be epi-Lipschitzian at a boundary point $x \in S$ if it can be represented near this point as the epigraph of a Lipschitzian function. This amounts to requiring that there be a neighborhood $U$ of $x$, a one-to-one linear mapping $\Lambda: \mathbb{R}^{n} \rightarrow \mathbb{R}^{n-1} \times \mathbb{R}$, and a locally Lipschitzian function $h: \mathbb{R}^{n-1} \rightarrow \mathbb{R}$ such that $U \cap S=U \cap \Lambda^{-1}$ (epi $\left.h\right)$. This local representation is shown in [26] to have a translation in terms of neighborhoods. Through adaptations of this translation, Rockafellar [27] extended the notion to sets in general locally convex vector spaces and introduced the concept of directionally Lipschitzian functions. Among several strong results of [26], it has been proved that, in the finite dimensional setting, the epi-Lipschitzian property of the set $S$ at $x$ is equivalent to the nonemptiness of its Clarke tangent cone at $x$. We emphasize (see [26, p. 148]) that such a characterization does not hold in infinite dimensional spaces. To illustrate how Lipschitz-like properties are significant in variational analysis, we recall some of the crucial results involving them.

*Received by the editors October 16, 2008; accepted for publication (in revised form) November 19, 2009; published electronically February 5, 2010. This research was partially supported by FONDECYT project 1080173 and Programa Basal CMM U. de Chile.

http://www.siam.org/journals/siopt/20-4/73827.html

${ }^{\dagger}$ Universidad de Chile, Centro de Modelamiento Matemático (CNRS UMI 2807) and Departamento de Ingeniería Matemática, Casilla 170/3, Correo 3, Santiago, Chile (rcorrea@dim.uchile.cl).

${ }^{\ddagger}$ Universidad Técnica Federico Santa María, Departamento de Matemática, Avda. España 1680, Valparaíso, Chile (pedro.gajardo@usm.cl).

§Université de Montpellier II, Département des Sciences Mathématiques, Place Eugène Bataillon, 34095 Montpellier Cedex 5, France (thibault@math.univ-montp2.fr). 
In [28] Rockafellar established, under a certain qualification condition, a subdifferential calculus rule for the sum of two functions, with the Clarke subdifferential, when one of the involved functions is directionally Lipschitzian. The same paper [28], invoking the epi-Lipschitzian property of sets, also established a rule for estimating the Clarke tangent cone of the intersection of two sets. Note that Rockafellar had already used this property in [26] for obtaining a rule in the estimation of the normal cone of the intersection of finitely many sets. Such calculus rules for functions and sets yield (see [28]) to general optimality conditions with Lagrange multipliers for optimization problems.

Treiman [35] proved that for closed epi-Lipschitzian sets of Banach spaces the Clarke tangent cone is the limit inferior of Boulingand contingent cones at neighboring points. Such an equality (known to be important in the viability theory of differential inclusions) was previously established by Cornet [9] and Penot [24] for any closed set of a finite dimensional space. Motivated by this formula in the infinite dimensional setting and by the above Rockafellar characterization of the epi-Lipschitzian property via the Clarke tangent cone, Borwein and Strójwas introduced some years later in [6] the notion of compactly epi-Lipschitzian sets. The class of such sets is much larger than the one defined by Rockafellar, and the property holds for every closed set in a finite dimensional normed space. In contrast to the epi-Lipschitzian predecessor concept, it makes it possible to recapture much of the detailed information available in finite dimensions. Moreover, a normed space is finite dimensional when every closed set is compactly epi-Lipschitzian. In the same work [6] it was shown that the equality between the Clarke tangent cone and the lower limit of Bouligand tangent cones still holds for compactly epi-Lipschitzian sets, unifying in this way the related results mentioned above and providing (in the infinite dimensional context) a new large class of sets for which the equality is fulfilled.

In the context of extended real valued lower semicontinuous (lsc) functions, the concept introduced by Borwein and Strójwas was translated by Jourani and Thibault in [19], where they provided a sum calculus rule for the Ioffe geometric subdifferential (see [15] for the definition) when one of the two functions is compactly epi-Lipschitzian. The same authors in [18] and Ioffe in [17] showed, for such sets in general Banach spaces, the equivalence between weak-star convergence to zero and strong convergence to zero of nets of geometric normal vectors at neighboring points. Earlier results along this line with Fréchet normal vectors are due to Loewen [21]. This convergence result allowed the authors of [19] and [17] to show point-based criteria for metric regularity of mappings or set-valued mappings from a Banach space into another space and to extend Lagrange optimality conditions of [15] with Ioffe's subdifferential under fewer assumptions on the data of the problem. We also refer to [22, 23], where it is proved that for Asplund spaces it is enough to require the graphical convergence property for sequences, instead of nets, of Fréchet normal vectors.

For the particular case of convex sets, Borwein, Lucet, and Mordukhovich showed in [4] the equality of the relative interior and the quasi-relative interior of compactly epi-Lipschitzian sets. The result is applied to the duality theorem given by Borwein and Lewis [5].

In view of the relevance illustrated above of directionally and compactly Lipschitzian properties to optimization and variational analysis, several authors have been interested in providing characterizations of these properties. In [28] Rockafellar established a characterization of directionally Lipschitzian behavior for convex functions, linking this property with the nonemptiness of the interior of the (effective) domains of the convex functions. 
The first characterization of the directionally Lipschitzian property for general lsc functions was given by Treiman in [36]. He showed, using the Bishop-Phelps technique, the equivalence between the directionally Lipschitzian property and a certain boundedness-like behavior of the Clarke subdifferential and also of the lower Dini directional derivative. The subdifferential characterization given by Treiman was extended by Thibault and Zlateva in [34] for any subdifferential satisfying the Zagrodny's mean value theorem (see [37, 33, 32]). In [11] Correa, Gajardo, and Thibault established the same characterization for any subdifferential or operator satisfying the representation formula of the Clarke subdifferential (see [29, 7, 20]).

For the compactly epi-Lipschitzian property of functions, Ioffe [16] gave a characterization in terms of his geometric subdifferential. The equivalence is related to some mixed boundedness property of the subdifferential. For a convex set, Borwein, Lucet, and Mordukhovich showed in [4] the equivalence between the compactly epiLipschitzian property and several conditions related to the interior (resp., relative interior) of the set $S+K$, the Minkowski sum of $S$, and some compact set $K$. They also derived similar characterizations of compactly epi-Lipschitzian behavior for convex functions.

The objective of this work is twofold. First, we aim to obtain equivalences for various Lipschitz-like properties in a unified way. Second, we intend to follow an approach allowing us to provide also several new characterizations as well as some new Lipschitz-like properties. The idea is to introduce a general notion that we call the $K$ directionally Lipschitzian property which covers for functions the concepts of locally Lipschitzian, directionally Lipschitzian, and compactly epi-Lipschitzian behaviors, and which covers for sets the epi-Lipschitzian and compactly epi-Lipschitzian concepts. The main tools for this purpose are the multidirectional mean value inequalities that we state for lower Dini directional derivatives. Multidirectional mean value inequalities were first discovered in a subdifferential version by Clarke and Ledyaev [8] in the context of Hilbert spaces. The extension of multidirectional mean value inequalities to general Banach spaces (with various classes of subdifferentials) is due to Aussel, Corvellec, and Lassonde [1], and the case of spaces with smooth bump functions is studied in Zhu [38]. The versions used in this paper are adaptations to lower Dini directional derivatives, which have been given in [12].

The characterizations of the $K$ directionally Lipschitzian property that we provide in this paper are, for a function $f$, related to some mixed boundedness property of the lower Dini directional derivative of $f$ and, for a set $S$, related to the interior of the Minkowski sum of the Bouligand tangent cone of $S$ and some compact set.

The paper is organized as follows. In the next section we recall the concepts of lower Dini directional derivatives, and we state the multidirectional mean value inequalities related to those directional derivatives. In section 3 we define the property of $K$ directionally Lipschitzian behavior of a function and establish relationships between this property and a certain mixed boundedness of lower Dini directional derivatives of the function. It is shown that the directional characterization of the directionally Lipschitzian property of functions given by Treiman [36] is recovered. Section 4 provides tangential characterizations, in terms of Bouligand tangent cones, of $K$ directionally Lipschitzian sets. In section 5, we study the particular case where convexity is present, providing in this way additional characterizations of convex functions or sets which are $K$ directionally Lipschitzian. The results of that section encompass characterizations given by Rockafellar [28] for the directionally Lipschitzian property of convex functions, and given by Borwein, Lucet, and Mordukhovich [4] for the compactly epiLipschitzian property of convex sets. Finally, we provide an application to the study 
of a Lipschitz-like property for the optimal value function of a certain optimization problem.

For characterizations of $K$ Lipschitz-like properties of functions in terms of subdifferentials which are not (necessarily) characterized by generalized directional derivatives as support functions, we refer to paper [10] which is a continuation of this one.

2. Preliminaries. Throughout the paper, unless otherwise stated, $(X,\|\cdot\|)$ stands for a real Banach space, $X^{*}$ for its topological dual, and $\langle\cdot, \cdot\rangle$ for the duality pairing.

For any lsc function $f: X \longrightarrow \mathbb{R} \cup\{+\infty\}$ and $\bar{x} \in \operatorname{dom} f:=\{x \in X: f(x)<$ $+\infty$, we begin by recalling some well-known concepts in variational analysis (see [22] and [31]).

The lower Dini directional derivative $d^{-} f(\bar{x} ; v)$ in a direction $v \in X$ is defined by

$$
d^{-} f(\bar{x} ; v)=\liminf _{\substack{v^{\prime} \rightarrow v \\ t \rightarrow 0^{+}}} t^{-1}\left[f\left(\bar{x}+t v^{\prime}\right)-f(\bar{x})\right] .
$$

A useful variant of this derivative in some Banach spaces is the sequential weak lower Dini directional derivative

$$
d_{w}^{-} f(\bar{x} ; v)=\inf _{\substack{v_{n} \rightarrow v \\ t_{n} \rightarrow 0^{+}}} \liminf _{n \rightarrow \infty} t_{n}^{-1}\left[f\left(\bar{x}+t_{n} v_{n}\right)-f(\bar{x})\right],
$$

where the weak topology is used in the notation $v_{n} \rightarrow \bar{v}$ and where the infimum is taken over all sequences $\left(v_{n}\right)_{n}$ and $\left(t_{n}\right)_{n}$ converging, respectively, to $v$ and $0^{+}$. Obviously, one has

$$
d_{w}^{-} f(\bar{x} ; \bar{v}) \leq d^{-} f(\bar{x} ; \bar{v}) .
$$

Recalling that the epigraph of $f$ is the set

$$
\text { epi } f:=\{(x, r) \in X \times \mathbb{R}: f(x) \leq r\},
$$

and the indicator function of a set $S \subset Y$ is defined for all $y$ in the space $Y$ by

$$
\Psi_{S}(y)= \begin{cases}0 & \text { if } y \in S \\ +\infty & \text { if } y \notin S\end{cases}
$$

it is easily seen that for all $\bar{x} \in \operatorname{dom} f$ and for $(\bar{v}, s) \in X \times \mathbb{R}$ one has

$$
\begin{aligned}
& d^{-} \Psi_{\text {epi } f}(\bar{x}, f(\bar{x}) ; \bar{v}, s)=\Psi_{\text {epi } d^{-} f(\bar{x} ; \cdot)}(\bar{v}, s) ; \\
& f(\bar{x}) \leq \beta \Rightarrow\left\{\begin{array}{l}
\Psi_{\text {epi } f}(\bar{x}+\bar{v}, \beta+s) \leq \Psi_{\text {epi } f}(\bar{x}+\bar{v}, f(\bar{x})+s), \\
d^{-} \Psi_{\text {epi } f}(\bar{x}, \beta ; \bar{v}, s) \leq d^{-} \Psi_{\text {epi } f}(\bar{x}, f(\bar{x}) ; \bar{v}, s) .
\end{array}\right.
\end{aligned}
$$

For an extended valued function $\phi$ on $X$ and a nonempty subset $S \subset X$ we define the limit of infimums of $\phi$ over enlargements of $S$ by

$$
\operatorname{linf}_{v \in S} \phi(v):=\lim _{\delta \rightarrow 0^{+}} \inf _{v \in S+\delta B_{X}} \phi(v)=\sup _{\delta>0} \inf _{v \in S+\delta B_{X}} \phi(v),
$$

where $B_{X}$ denotes the open unit ball in $X$ centered at the origin. 
The equality of the following lemma under the compactness of $S$ has been previously observed in Proposition 3.2 of [1]. For the convenience of the reader we give a simple proof.

LEMMA 2.1. If $S \subset X$ is a compact set and $\phi: X \longrightarrow \mathbb{R} \cup+\infty$ is lsc, then

$$
\operatorname{linf}_{v \in S} \phi(v)=\inf _{v \in S} \phi(v) .
$$

Proof. Evidently one has

$$
\operatorname{linf}_{v \in S} \phi(v) \leq \inf _{v \in S} \phi(v)
$$

To prove the opposite inequality we may suppose that $\operatorname{linf}_{v \in S} \phi(v)<+\infty$. Fix any real number $r>\operatorname{linf}_{v \in S} \phi(v)$. Then, by (2.6), for each $n \in \mathbb{N}$ there exist $v_{n} \in S$ and $u_{n} \in(1 / n) B_{X}$ such that $\phi\left(v_{n}+u_{n}\right)<r$. Since $S$ is compact, we can take a subsequence of $v_{n}+u_{n}$ converging to some $\bar{v} \in S$. From the lower semicontinuity of $\phi$ we obtain

$$
\inf _{v \in S} \phi(v) \leq \phi(\bar{v}) \leq r
$$

So we conclude that $\inf _{v \in S} \phi(v) \leq \operatorname{linf}_{v \in S} \phi(v)$.

Our main variational tools in this work are the multidirectional mean value inequalities that we give in Theorem 2.2 below. The theorem was first established in its subdifferential version (in fact, with the smallest known subdifferential, which is the proximal one) by Clarke and Ledyaev (see [8]) in the context of Hilbert spaces. Its extension to general Banach spaces (with various classes of subdifferentials) is due to Aussel, Corvellec, and Lassonde [1]. The versions below are adaptations to Dini directional derivatives which have been given in [12].

In the statement of the theorem we denote by $d_{S}(x)$ the distance from $x$ to a set $S \subset X$, i.e, $d_{S}(x):=\inf _{y \in S}\|x-y\|$.

TheOREM 2.2. Let $C \subset X$ be a nonempty closed convex set, $f: X \longrightarrow \mathbb{R} \cup\{+\infty\}$ a lsc function, and $a \in \operatorname{dom} f$. Assume that for the drop

$$
D=[a, C]:=\{\lambda a+(1-\lambda) c: \lambda \in[0,1], \quad c \in C\}
$$

there exists some $s>0$ such that $f$ is bounded from below over the enlargement $D+s B_{X}$ of $D$. Then, for every real number $\rho \leq \operatorname{linf}_{z \in C} f(z)$, the following properties hold:

(a) There exists a sequence $\left\{x_{n}\right\} \subset \operatorname{dom} f$ such that $\mathrm{d}_{D}\left(x_{n}\right) \rightarrow 0$ and

$$
d^{-} f\left(x_{n} ; c-a\right) \geq \rho-f(a)-(1 / n)\|c-a\|-1 / n \quad \forall c \in C, \forall n .
$$

(b) If $X$ is an Asplund space, then there exists a sequence $\left\{x_{n}\right\} \subset \operatorname{dom} f$ such that $\mathrm{d}_{D}\left(x_{n}\right) \rightarrow 0$ and

$$
d_{w}^{-} f\left(x_{n} ; c-a\right) \geq \rho-f(a)-(1 / n)\|c-a\|-1 / n \quad \forall c \in C, \forall n .
$$

Proof. The relation (a) can be established following the proof of Theorem 6.1 in [1]. For the second inequality see [12, Proposition 1]. 
3. Directional characterizations. We begin this section by introducing, for any subset $K \subset X$, the concept of $K$ directionally Lipschitzian functions. Throughout, for $x \in X$ and $r>0$ we denote by $B(x, r)$ the open ball of $X$ centered at $x$ with radius $r$.

Definition 3.1. We say that a lsc function $f: X \longrightarrow \mathbb{R} \cup\{+\infty\}$ is $K$ directionally Lipschitzian at $\bar{x} \in \operatorname{dom} f$, for some subset $K \subset X$, if there exists $\beta \in \mathbb{R}$ such that

$$
\limsup _{\substack{x \rightarrow f \bar{x} \\ t \rightarrow 0^{+} \\ b \rightarrow 0}} \operatorname{linf}_{v \in K} t^{-1}[f(x+t v+t b)-f(x)]<\beta
$$

or, equivalently, if there exist $\beta \in \mathbb{R}$ and $r>0$ such that

$$
\operatorname{linf}_{v \in K} t^{-1}[f(x+t v+t b)-f(x)]<\beta
$$

for all $t \in] 0, r], b \in r B_{X}$, and

$$
x \in B_{f}(\bar{x}, r):=\left\{x \in X: x \in \bar{x}+r B_{X}, \quad|f(x)-f(\bar{x})|<r\right\} .
$$

The above notation $x \rightarrow_{f} \bar{x}$ means that $(x, f(x)) \rightarrow(\bar{x}, f(\bar{x}))$. Observe that the expression whose linf is taken in (3.1) and (3.2) is well defined because $f(x)$ is finite for $x \in B_{f}(\bar{x}, r)$.

When $f$ is $K$ directionally Lipschitzian at $\bar{x}$, obviously it is also $K^{\prime}$ directionally Lipschitzian at $\bar{x}$ for any set $K^{\prime} \supset K$. It is also easily seen that $f$ is $K$ directionally Lipschitzian at $\bar{x}$ if and only if it is $\rho K$ directionally Lipschitzian for any $\rho>0$.

REMARK 3.2. We note that the notion of $K$ directionally Lipschitzian behavior recovers some well-known concepts in variational analysis.

- Obviously a lsc function is locally Lipschitzian at a point of its effective domain if and only if it is $\{0\}$ directionally Lipschitzian at that point.

Recall (see $[27,6,19]$ ) that a lsc function $f: X \rightarrow \mathbb{R} \cup\{+\infty\}$ is directionally Lipschitzian (resp., compactly epi-Lipschitzian) at $\bar{x} \in \operatorname{dom} f$ if and only if there exists a vector $\bar{v}$ (resp., a compact set $K$ ) of $X$, a real number $\beta$, and a positive number $r$ such that

$$
t^{-1}[f(x+t \bar{v}+t b)-f(x)]<\beta \quad\left(\text { resp. }, \inf _{v \in K} t^{-1}[f(x+t v+t b)-f(x)]<\beta\right)
$$

for all $x \in B_{f}(\bar{x}, r), b \in r B_{X}$, and $\left.t \in\right] 0, r[$. So, according to (3.2),

- a lsc function is directionally Lipschitzian at a point of its effective domain (see [27]) if and only if it is $\{\bar{v}\}$ directionally Lipschitzian at that point for some $\bar{v} \in X$;

- a lsc function is compactly epi-Lipschitzian at a point of its effective domain (see [6, 19]) if and only if it is $K$ directionally Lipschitzian at that point for some compact set $K \subset X$.

Finally, we observe that every lsc function is $\bar{B}_{X}$ (the closed unit ball) directionally Lipschitzian at any point of its effective domain, and therefore every lsc function on $X$ is compactly epi-Lipschitzian at any point of its effective domain whenever $X$ is a finite dimensional space. In fact, the compactly epi-Lipschitzian property of any lsc function $f$ on $X$ characterizes the fact that $X$ is finite dimensional since, taking as $f$ the indicator function of the singleton $\{0\}$, the second inequality of (3.3) entails that for the positive number $r$ and the compact set $K$ of (3.3) one has $r B_{X} \subset K$, and hence the compact set $K$ is a neighborhood of the origin. 
The following proposition provides some mixed upper boundedness property of the lower Dini directional derivative of a $K$ directionally Lipschitzian function.

Proposition 3.3. Let $f: X \longrightarrow \mathbb{R} \cup\{+\infty\}$ be a lsc $K$ directionally Lipschitzian function at $\bar{x} \in \operatorname{dom} f$. If $K \subset X$ is compact, then

$$
\sup _{b \in r B_{X}} \inf _{v \in K} d^{-} f(x ; v+b) \leq \beta
$$

for all $x \in B_{f}(\bar{x}, r)$, where $r>0$ and $\beta \in \mathbb{R}$ are as given by (3.2) in Definition 3.1.

Proof. Take $r>0$ and $\beta \in \mathbb{R}$ given by (3.2) and fix $x \in B_{f}(\bar{x}, r), b \in r B_{X}$, and $t_{n} \rightarrow 0^{+}$. Since $K$ is compact, from Lemma 2.1 and inequality (3.2), for $n$ large enough there exists $v_{n} \in K$ such that

$$
t_{n}^{-1}\left[f\left(x+t_{n} v_{n}+t_{n} b\right)-f(x)\right]<\beta .
$$

Taking a subsequence of $\left(v_{n}\right)$ converging to some $v=v\left(x,\left\{t_{n}\right\}, b\right) \in K$ and passing to the limit inferior in the above inequality, one obtains

$$
d^{-} f(x ; v+b) \leq \beta,
$$

which proves the desired result.

When $K$ is weakly compact, a similar mixed upper boundedness property holds with the weak sequential lower Dini directional derivative defined in (2.2).

Proposition 3.4. Let $f: X \longrightarrow \mathbb{R} \cup\{+\infty\}$ be a lsc function which is $K$ directionally Lipschitzian at $\bar{x} \in \operatorname{dom} f$. If $K \subset X$ is weakly compact, then

$$
\sup _{b \in r B_{X}} \inf _{v \in K} d_{w}^{-} f(x ; v+b) \leq \beta
$$

for all $x \in B_{f}(\bar{x}, r)$, where $r>0$ and $\beta \in \mathbb{R}$ are as given by (3.2) in Definition 3.1.

Proof. Fix $r>0$ and $\beta \in \mathbb{R}$ satisfying (3.2). For $x \in B_{f}(\bar{x}, r), b \in r B_{X}$, and $t_{n} \rightarrow 0^{+}$we have, for $n$ large enough,

$$
\operatorname{linf}_{v \in K} t_{n}^{-1}\left[f\left(x+t_{n} v+t_{n} b\right)-f(x)\right]<\beta .
$$

According to (2.6), for such integers $n$, choose $v_{n} \in K$ and $u_{n} \in(1 / n) B_{X}$ such that

$$
t_{n}^{-1}\left[f\left(x+t_{n}\left(v_{n}+u_{n}\right)+t_{n} b\right)-f(x)\right]<\beta .
$$

We use now the Eberlein-Šmulian theorem (see, for example, [14, Theorem V.6.1]) in order to take a subsequence of $\left(v_{n}\right)$ converging weakly to some $v=v\left(x,\left\{t_{n}\right\}, b\right) \in K$, and hence

$$
\begin{gathered}
d_{w}^{-} f(x ; b+v)=\inf _{\substack{w_{n} \rightarrow b+v \\
s_{n} \rightarrow 0^{+}}} \liminf _{n \rightarrow \infty} s_{n}^{-1}\left[f\left(x+s_{n} w_{n}\right)-f(x)\right] \\
\leq \liminf _{n \rightarrow \infty} t_{n}^{-1}\left[f\left(x+t_{n}\left(v_{n}+u_{n}\right)+t_{n} b\right)-f(x)\right] \leq \beta
\end{gathered}
$$

In order to establish the converse of Propositions 3.3 and 3.4 (without any compactness assumptions on $K$ ) we give the following lemma.

Lemma 3.5. A lsc function $f: X \longrightarrow \mathbb{R} \cup\{+\infty\}$ is $K$ directionally Lipschitzian at $\bar{x} \in \operatorname{dom} f$ if and only if the indicator function of the epigraph of $f$ is $K \times\left\{\beta^{\prime}\right\}$ directionally Lipschitzian at $(\bar{x}, f(\bar{x}))$ for some $\beta^{\prime} \in \mathbb{R}$. 
Proof. Suppose that $f$ is $K$ directionally Lipschitzian at $\bar{x}$, and fix $\beta \in \mathbb{R}$ and $r>0$ given by (3.2). Then, for all $\left.x \in B_{f}(\bar{x}, r), t \in\right] 0, r\left[, b \in r B_{X}\right.$, and $\delta>0$ there exists $v_{\delta} \in K+\delta B_{X}$ such that

$$
f\left(x+t b+t v_{\delta}\right)<f(x)+t \beta .
$$

By lower semicontinuity of $f$ at $\bar{x}$ choose some $\left.r^{\prime} \in\right] 0, r[$ such that $f(x)>f(\bar{x})-r$ for all $x \in B\left(\bar{x}, r^{\prime}\right)$. Then, we see that for all $(x, s) \in$ epi $f \cap B\left((\bar{x}, f(\bar{x})), r^{\prime}\right)$, $\left.\left(b, b^{\prime}\right) \in r B_{X} \times\right]-r, r[, t \in] 0, r\left[\right.$, and $\delta>0$ we have $x \in B_{f}(\bar{x}, r)$, and hence there exists $\left(v_{\delta}, p_{\delta}\right) \in(K \times\{\beta+r\})+\left(\delta B_{X} \times\right]-\delta, \delta[)$ (in fact, we can choose $p_{\delta}=\beta+r$ ) such that

$$
f\left(x+t b+t v_{\delta}\right) \leq s+t b^{\prime}+t p_{\delta},
$$

which is equivalent to the inequality

$$
t^{-1}\left[\Psi_{\text {epi } f}\left(x+t b+t v_{\delta}, s+t b^{\prime}+t p_{\delta}\right)-\Psi_{\text {epi } f}(x, s)\right] \leq 0,
$$

and therefore

$$
\operatorname{linf}_{(v, p) \in K \times\{\beta+r\}} t^{-1}\left[\Psi_{\text {epi } f}\left(x+t b+t v, s+t b^{\prime}+t p\right)-\Psi_{\text {epi } f}(x, s)\right] \leq 0,
$$

concluding that, for $\beta^{\prime}=\beta+r$, the function $\Psi_{\text {epi } f}$ is $K \times\left\{\beta^{\prime}\right\}$ epi-Lipschitzian at $(\bar{x}, f(\bar{x}))$.

Let us now suppose that $\Psi_{\text {epi } f}$ is $K \times\left\{\beta^{\prime}\right\}$ directionally Lipschitzian at $(\bar{x}, f(\bar{x}))$. Thus, there exists $r>0$ such that for all $(x, s) \in$ epi $f \cap B((\bar{x}, f(\bar{x})), r)$, $\left.\left(b, b^{\prime}\right) \in r B_{X} \times\right]-r, r[$, and $t \in] 0, r[$ one has

$$
\operatorname{linf}_{(v, p) \in K \times\left\{\beta^{\prime}\right\}} t^{-1}\left[\Psi_{\text {epi } f}\left(x+t b+t v, s+t b^{\prime}+t p\right)-\Psi_{\text {epi } f}(x, s)\right]<+\infty .
$$

This implies that, for all $\delta>0$, there exists $v_{\delta} \in K+\delta B_{X}$ and $\left.\beta_{\delta} \in\left\{\beta^{\prime}\right\}+\right]-r, r[$ such that

$$
f\left(x+t b+t v_{\delta}\right) \leq s+t b^{\prime}+t \beta_{\delta},
$$

and hence, in particular, for any $x \in B_{f}(\bar{x}, r)$ with the choice of $s=f(x)$ we can obtain (3.2) with $\beta=\beta^{\prime}+2 r$.

The following result establishes a converse of Proposition 3.3 via the multidirectional mean value inequality (2.7).

Proposition 3.6. Let $f: X \longrightarrow \mathbb{R} \cup\{+\infty\}$ be a lsc function, $\bar{x} \in \operatorname{dom} f$, and let $K \subset X$ be a closed bounded subset. Assume that there exist $r>0$ and $\beta \in \mathbb{R}$ such that

$$
\sup _{b \in r B_{X}} \inf _{v \in K} d^{-} f(x ; v+b) \leq \beta
$$

for all $x \in B_{f}(\bar{x}, r)$. Then, $f$ is $\overline{\mathrm{co}} K$ directionally Lipschitzian at $\bar{x}$, where $\overline{\mathrm{co}} K$ denotes the closed convex hull of $K$.

Proof. We put $K^{\prime}=\overline{\mathrm{co}} K$. We first suppose $f$ to be continuous on $\operatorname{dom} f$ with respect to the induced topology on $\operatorname{dom} f$. Let $\bar{\nu}>0$ be such that $f$ is bounded from below on $B(\bar{x}, \bar{\nu})$. For any $\eta>0$ we denote by $\xi(\eta) \in] 0, \eta[$ a positive number such that

$$
B(\bar{x}, \xi(\eta)) \cap \operatorname{dom} f \subset B_{f}(\bar{x}, \eta)
$$


Without loss of generality we assume $r<\bar{\nu}$. Let $\left.r^{\prime} \in\right] 0, \xi(r) / 2[$ be such that

$$
\left.B\left(\bar{x}, r^{\prime}\right)+\right] 0, r^{\prime}\left[r^{\prime} B_{X}+\right] 0, r^{\prime}\left[K^{\prime} \subset B(\bar{x}, \xi(r) / 2) .\right.
$$

Fix $\left.x \in B_{f}\left(\bar{x}, r^{\prime}\right), t \in\right] 0, r^{\prime}\left[\right.$, and $b \in r^{\prime} B_{X}$. We define the closed convex set $C=$ $x+t b+t K^{\prime}$ and put $D=[x, C]$. Observe that

$$
C \subset D \subset B(\bar{x}, \xi(r) / 2) \subset B(\bar{x}, \bar{\nu} / 2),
$$

and hence the boundedness from below of $f$ over $B(\bar{x}, \bar{\nu})$ yields that $f$ is bounded from below over $D+(\bar{\nu} / 2) B_{X}$. Further, this ensures us in particular that $\operatorname{linf}_{z \in C} f(z)>-\infty$. For all $\delta>0$, since

$$
\inf _{z \in C+\delta t B_{X}} f(z) \leq \operatorname{linf}_{z \in C} f(z) \text { and } \operatorname{linf}_{z \in C} f(z)>-\infty
$$

we have that for any $\alpha>0$ and $\delta>0$ there exist $v^{\prime \prime} \in K^{\prime}+\delta B_{X}$ (depending on $\alpha$ and $\delta$ ) such that

$$
f\left(x+t b+t v^{\prime \prime}\right)-\alpha t \leq \operatorname{linf}_{z \in C} f(z) .
$$

(1) Fix $\alpha>0$ and $\delta>0$. We first show that $f\left(x+t b+t v^{\prime \prime}\right)$ is finite. Consider any real number $\rho \leq f\left(x+t b+t v^{\prime \prime}\right)$ (such numbers exist since $f\left(x+t b+t v^{\prime \prime}\right)>-\infty$ ). Taking into account the boundedness from below of $f$ over $D+(\bar{\nu} / 2) B_{X}$ and the fact that $f(x)$ is finite because $x \in B_{f}\left(\bar{x}, r^{\prime}\right)$, we may apply the mean value inequality (2.7). This provides, for the real number $\rho-\alpha t$ satisfying $\rho-t \alpha \leq \operatorname{linf}_{z \in C} f(z)$ (see (3.6)), the existence, for each integer $n$, of some $x_{n} \in \operatorname{dom} f$ with $\mathrm{d}_{D}\left(x_{n}\right) \rightarrow 0$ such that

$$
d^{-} f\left(x_{n} ; t b+t v\right) \geq \rho-t \alpha-f(x)-t\|v+b\| / n-1 / n
$$

for all $v \in K^{\prime}$. This yields for all $v \in K^{\prime}$ and all integers $n$

$$
t d^{-} f\left(x_{n} ; b+v\right) \geq \rho-t \alpha-f(x)-t \mu / n-1 / n,
$$

where $\mu=\sup _{v \in K^{\prime}}\|v\|+r^{\prime}$ is finite due to the boundedness of $K^{\prime}$. Since $\mathrm{d}_{D}\left(x_{n}\right)<$ $\xi(r) / 2$ for $n$ large enough, by (3.5), we have for such integers $n$

$$
x_{n} \in B(\bar{x}, \xi(r)) \cap \operatorname{dom} f \subset B_{f}(\bar{x}, r) .
$$

Then by (3.7) and assumption (3.4) we have in particular

$$
t \beta \geq \rho-t \alpha-f(x)-t \mu-1 .
$$

This shows that $f\left(x+t b+t v^{\prime \prime}\right)$ is finite.

(2) Since $f(x+t b+t v)$ is finite, we may take $\rho=f(x+t b+t v) \in \mathbb{R}$ and proceed with arguments as in (1). For the appropriate sequence $\left(x_{n}\right)$ and for $n$ large enough, according to (3.7) and our assumption (3.4) we have

$$
\beta \geq d^{-} f\left(x_{n} ; b+v\right) \geq t^{-1}\left[f\left(x+t b+t v^{\prime \prime}\right)-f(x)\right]-\mu / n-1 /(t n)-\alpha,
$$

and hence

$$
\beta \geq \inf _{v^{\prime} \in K^{\prime}+\delta B_{X}} t^{-1}\left[f\left(x+t b+t v^{\prime}\right)-f(x)\right]-\mu / n-1 /(t n)-\alpha .
$$


Taking the limit for $n \rightarrow \infty$ and then the limit for $\alpha \rightarrow 0^{+}$, we obtain

$$
\beta \geq \inf _{v^{\prime} \in K^{\prime}+\delta B_{X}} t^{-1}\left[f\left(x+t b+t v^{\prime}\right)-f(x)\right] .
$$

Therefore

$$
\beta \geq \sup _{\delta>0} \inf _{v^{\prime} \in K^{\prime}+\delta B_{X}} t^{-1}\left[f\left(x+t b+t v^{\prime}\right)-f(x)\right]=\operatorname{linf}_{v^{\prime} \in K^{\prime}} t^{-1}\left[f\left(x+t b+t v^{\prime}\right)-f(x)\right]
$$

for all $\left.x \in B_{f}\left(\bar{x}, r^{\prime}\right), t \in\right] 0, r^{\prime}\left[\right.$, and $b \in r^{\prime} B_{X}$. According to (3.2) we conclude that $f$ is $K^{\prime}$ directionally Lipschitzian at $\bar{x}$.

Let us prove now that (3.4) implies that, for some $\beta^{\prime} \in \mathbb{R}$ and some $r^{\prime}>0$, we have

$$
\sup _{\left.\left(b, b^{\prime}\right) \in r^{\prime} B_{X} \times\right]-r^{\prime}, r^{\prime}\left[\left(v, \beta^{\prime}\right) \in K^{\prime} \times\left\{\beta^{\prime}\right\}\right.} d^{-} \Psi_{\operatorname{epi} f}\left((x, s) ;\left(v+b, \beta^{\prime}+b^{\prime}\right)\right) \leq 0
$$

for all $(x, s) \in$ epi $f \cap B\left((\bar{x}, f(\bar{x})), r^{\prime}\right)$.

On the one hand, fixing $\gamma>0$, we obtain from (3.4) that for all $x \in B_{f}(\bar{x}, r)$, $\left.\left(b, b^{\prime}\right) \in r B_{X} \times\right]-r, r\left[\right.$ there exists $v \in K^{\prime}$ such that $d^{-} f(x ; v+b) \leq \beta+r+b^{\prime}+\gamma$, or equivalently, for $\beta^{\prime}:=\beta+\gamma+r$,

$$
\Psi_{\text {epi } d^{-} f(x ; \cdot)}\left(v+b, \beta^{\prime}+b^{\prime}\right)=0,
$$

which implies from (2.3) that

$$
d^{-} \Psi_{\text {epi } f}\left((x, f(x)) ;\left(v+b, \beta^{\prime}+b^{\prime}\right)\right)=0 .
$$

On the other hand, if $(x, s) \in$ epi $f$, one has from (2.5) that

$$
d^{-} \Psi_{\text {epi } f}\left((x, s) ;\left(v+b, \beta^{\prime}+b^{\prime}\right)\right) \leq d^{-} \Psi_{\text {epi } f}\left((x, f(x)) ;\left(v+b, \beta^{\prime}+b^{\prime}\right)\right) .
$$

Choose by lower semicontinuity of $f$ some $\left.r^{\prime} \in\right] 0, r[$ such that $f(x)>f(\bar{x})-r$ for all $x \in B\left(\bar{x}, r^{\prime}\right)$. Thus, we may see that for all $(x, s) \in$ epi $f \cap B\left((\bar{x}, f(\bar{x})), r^{\prime}\right)$ and for all $\left.\left(b, b^{\prime}\right) \in r^{\prime} B_{X} \times\right]-r^{\prime}, r^{\prime}\left[\right.$ there exists $\left(v, \beta^{\prime}\right) \in K^{\prime} \times\left\{\beta^{\prime}\right\}$ such that

$$
d^{-} \Psi_{\text {epi } f}\left((x, s) ;\left(v+b, \beta^{\prime}+b^{\prime}\right)\right) \leq 0,
$$

which means that (3.8) is satisfied. Since the function $\Psi_{\text {epi } f}$ is continuous on its effective domain with respect to the induced topology, from the first part of the proof we obtain that $\Psi_{\text {epi } f}$ is $K^{\prime} \times\left\{\beta^{\prime}\right\}$ directionally Lipschitzian at $(\bar{x}, f(\bar{x}))$, and by Lemma 3.5 we conclude that $f$ is $K^{\prime}$ directionally Lipschitzian at $\bar{x}$.

REMARK 3.7. The argument of part (1) above clarifies the fact that $f\left(x+t v^{\prime \prime}\right)$ in line 4 of page 64 of [12] is finite when the second member of inequality (14) in [12] is less than $+\infty$. This point has been omitted in the proof of Theorem 2 in [12].

In the case of Asplund spaces the multidirectional mean value inequality (2.8) may be used, instead of inequality (2.7), to establish a converse of Proposition 3.4.

Proposition 3.8. Let $f: X \longrightarrow \mathbb{R} \cup\{+\infty\}$ be a lsc function, $\bar{x} \in \operatorname{dom} f, K \subset X$ a closed and bounded subset, $r>0$, and $\beta \in \mathbb{R}$ such that

$$
\sup _{b \in r B_{X}} \inf _{v \in K} d_{w}^{-} f(x ; v+b) \leq \beta
$$

for all $x \in B_{f}(\bar{x}, r)$. If $X$ is an Asplund space, then $f$ is $\overline{\mathrm{co}} K$ directionally Lipschitzian at $\bar{x}$. 
Proof. The proof is very similar to that of Proposition 3.6 using the multidirectional mean value inequality (2.8) for $d_{w}^{-} f$.

From the above propositions we obtain the following theorem. It provides a characterization of $K$ directionally Lipschitzian functions in terms of the lower Dini directional derivative.

TheOREM 3.9. For any lsc function $f: X \longrightarrow \mathbb{R} \cup\{+\infty\}$ the following assertions hold:

(a) If $K \subset X$ is convex compact, then the function $f$ is $K$ directionally Lipschitzian at $\bar{x} \in \operatorname{dom} f$ if and only if there exist $r>0$ and $\beta \in \mathbb{R}$ such that

$$
\sup _{b \in r B_{X}} \inf _{v \in K} d^{-} f(x ; v+b) \leq \beta \quad \forall x \in B_{f}(\bar{x}, r) .
$$

(b) If $K \subset X$ is convex weakly compact and $X$ is an Asplund space, then the function $f$ is $K$ directionally Lipschitzian at $\bar{x} \in \operatorname{dom} f$ if and only if there exist $r>0$ and $\beta \in \mathbb{R}$ such that

$$
\sup _{b \in r B_{X}} \inf _{v \in K} d_{w}^{-} f(x ; v+b) \leq \beta \quad \forall x \in B_{f}(\bar{x}, r) .
$$

As a direct consequence of the above theorem we obtain characterizations for directionally Lipschitzian and compactly epi-Lipschitzian functions in the sense of [28] and [6] (see also [19]), respectively, in terms of the lower Dini directional derivatives. The characterization of the directionally Lipschitzian property in terms of the lower Dini directional derivative (part (i) in Corollary 3.10 below) was previously established by Treiman in [36, Theorem 6] using the technique of Bishop and Phelps [2].

To our knowledge, characterizations for directionally Lipschitzian behavior, in terms of the sequential weak lower Dini derivative, and characterizations for compactly epi-Lipschitzian functions given in the next corollary, are new. Then

Corollary 3.10. Let $f: X \longrightarrow \mathbb{R} \cup\{+\infty\}$ be a lsc function, and let $\bar{x} \in \operatorname{dom} f$.

(i) $f$ is directionally Lipschitzian at $\bar{x}$ if and only if there exist some $\bar{v} \in X$, $r>0$, and $\beta \in \mathbb{R}$ such that

$$
d^{-} f(x ; v) \leq \beta \quad \forall x \in B_{f}(\bar{x}, r), \quad v \in B(\bar{v}, r) .
$$

(ii) $f$ is compactly epi-Lipschitzian at $\bar{x} \in \operatorname{dom} f$ if and only if there exist a compact set $K \subset X, r>0$, and $\beta \in \mathbb{R}$ such that

$$
\sup _{b \in r B_{X}} \inf _{v \in K} d^{-} f(x ; v+b) \leq \beta \quad \forall x \in B_{f}(\bar{x}, r) .
$$

(iii) In the case when $X$ is Asplund, the directionally Lipschitzian and compactly epi-Lipschitzian properties of $f$ at $\bar{x}$ are also characterized as above but with $d_{w}^{-} f(x ; \cdot)$ in place of $d^{-} f(x ; \cdot)$.

Proof. Using (a) for (i) and (ii) (resp., (b) for (iii)) in Theorem 3.9, it is a direct consequence of the inequality $\inf _{\overline{c o} K} \leq \inf _{K}$ and the fact that $\overline{c o} K$ is compact when $K$ is compact (see, for example, [14, Theorem V.2.6]).

Although we will not go further with the usual Lipschitzian property, we emphasize that with $K=\{0\}$ in Theorem 3.9, we obtain that the lsc function $f$ on the general (resp., Asplund) Banach space $X$ is Lipschitzian near $\bar{x}$ if and only if there exist $r>0$ and $\beta \in \mathbb{R}_{+}$such that $d^{-} f(x ; v) \leq \beta$ (resp., $d_{w}^{-} f(x ; v) \leq \beta$ ) for all $x \in B_{f}(\bar{x}, r)$ and $v \in r \bar{B}_{X}$. See [36] for the characterization inequality with $d^{-} f(\cdot ; \cdot)$. 
4. Tangential characterizations. In this section, we will provide characterizations of Lipschitz-like properties for closed sets. Let us start first with the following definition.

Definition 4.1. We say that a closed set $S \subset X$ is $K$ directionally Lipschitzian at $\bar{x} \in S$, for some set $K \subset X$, if its indicator function $\Psi_{S}$ is $K$ directionally Lipschitzian at $\bar{x}$. This means that there exists $r>0$ such that for all $t \in] 0, r[$ one has

$$
S \cap B(\bar{x}, r)+\operatorname{tr} B_{X} \subset \bigcap_{\delta>0}\left(S-t\left(K+\delta B_{X}\right)\right)=\operatorname{cl}(S-t K) .
$$

REMARK 4.2. In the above definition notice that if $K \subset X$ is compact, one has

$$
\bigcap_{\delta>0}\left(S-t\left(K+\delta B_{X}\right)\right)=S-t K
$$

In fact, for this case $S-t K$ will be closed.

REMARK 4.3. From the above remark we note that the notion of $K$ directionally Lipschitzian behavior recovers two well-known concepts in variational analysis. Recall that a closed set $S$ is epi-Lipschitzian in the sense of [27] (resp., compactly epi-Lipschitzian in the sense of [6]) at $\bar{x} \in S$ when there exist a vector $\bar{v} \in X$ (resp., a compact set $K$ of $X$ ) and a real number $r>0$ such that

$$
\begin{gathered}
S \cap B(\bar{x}, r)+] 0, r[B(\bar{v}, r) \subset S \\
\left(r e s p ., S \cap B(\bar{x}, r)+t r B_{X} \subset S-t K \quad \forall t \in\right] 0, r[) .
\end{gathered}
$$

So, according to the above remark we have the following equivalences:

- A closed set is epi-Lipschitzian at one of its points if and only if it is $\{v\}$ directionally Lipschitzian at that point for some $v \in X$.

- A closed set is compactly epi-Lipschitzian at one of its points if and only if it is $K$ directionally Lipschitzian at that point for some compact set $K \subset X$.

Finally, we observe that every closed set is a $\bar{B}_{X}$ directionally Lipschitzian set, where $\bar{B}_{X}$ is the closed unit ball in $X$, and therefore, if $X$ is a finite dimensional space, every closed set of $X$ is compactly epi-Lipschitzian. In fact, arguing as in the previous section for lsc functions, we see that the latter property characterizes the finite dimensional vector spaces in the sense that a Banach space is finite dimensional if and only if every one of its closed sets is compactly epi-Lipschitzian. In fact, $X$ is finite dimensional whenever $\{0\}$, and a fortiori every singleton, is a compactly epi-Lipschitzian set.

Now we will apply Theorem 3.9 in order to obtain tangential characterizations for $K$ directionally Lipschitzian sets. With this purpose we recall that the Bouligand tangent cone to $S \subset X$ at $x \in S$ is defined by

$$
\begin{aligned}
T_{B}(S ; x) & :=\operatorname{Limsup}_{t \rightarrow 0^{+}} t^{-1}(S-x) \\
& =\left\{v \in X: \exists t_{n} \rightarrow 0^{+}, \exists v_{n} \rightarrow v \text { with } v_{n} \in t_{n}^{-1}(S-x)\right\},
\end{aligned}
$$


and the weak sequential Bouligand tangent cone to $S$ at $x \in S$ is given by

$$
\begin{aligned}
T_{B}^{w}(S ; x): & =w-\underset{t \rightarrow 0^{+}}{\operatorname{Limsup}} t^{-1}(S-x) \\
& =\left\{v \in X: \exists t_{n} \rightarrow 0^{+}, \exists v_{n} \rightarrow v \text { with } v_{n} \in t_{n}^{-1}(S-x)\right\},
\end{aligned}
$$

where $v_{n} \rightarrow v$ stands for the convergence with respect to the weak topology.

From the above definitions, it is straightforward to see that

$$
\begin{aligned}
\Psi_{T_{B}(S ; \bar{x})}(\bar{v}) & =d^{-} \Psi_{S}(\bar{x} ; \bar{v}), \\
\Psi_{T_{B}^{w}(S ; \bar{x})}(\bar{v}) & =d_{w}^{-} \Psi_{S}(\bar{x} ; \bar{v}) .
\end{aligned}
$$

Translating Proposition 3.6 with indicator functions yields the following result.

Proposition 4.4. Let $S \subset X$ be a nonempty closed set, and let $\bar{x} \in S$. If one of the following two assertions is satisfied for some closed bounded set $K \subset X$ and some $r>0$, then $S$ is $\overline{\mathrm{co}} K$ directionally Lipschitzian at $\bar{x}$ :

(a)

$$
r B_{X} \subset T_{B}(S ; x)-K \quad \forall x \in B(\bar{x}, r) \cap S .
$$

(b) $X$ is an Asplund space and

$$
r B_{X} \subset T_{B}^{w}(S ; x)-K \quad \forall x \in B(\bar{x}, r) \cap S .
$$

Proof. The proof is direct from Proposition 3.6 and equalities (4.1) and (4.2).

In a similar way, using Theorem 3.9, we obtain the following characterization.

THEOREM 4.5. For any closed set $S \subset X$ the following assertions hold:

(a) The set $S$ is $K$ directionally Lipschitzian at $\bar{x} \in S$ for some convex compact set $K \subset X$ if and only if there exists $r>0$ such that

$$
r B_{X} \subset T_{B}(S ; x)-K \quad \forall x \in B(\bar{x}, r) \cap S .
$$

(b) If $X$ is Asplund, then $S$ is $K$ directionally Lipschitzian at $\bar{x} \in S$ with $K$ convex weakly compact if and only if there exists $r>0$ such that

$$
r B_{X} \subset T_{B}^{w}(S ; x)-K \quad \forall x \in B(\bar{x}, r) \cap S .
$$

Proof. The proof is direct from Theorem 3.9 together with equalities (4.1) and (4.2).

As a consequence, we obtain the following new characterizations of epi-Lipschitzian and compactly epi-Lipschitzian sets in terms of tangent cones, which follow directly from Theorem 4.5.

Corollary 4.6. Let $S$ be a closed subset of $X$ and $\bar{x} \in S$. Then the following hold:

(a) The set $S$ is epi-Lipschitzian (resp., compactly epi-Lipschitzian) at $\bar{x}$ if and only if there exist some $\bar{v} \in X$ (resp., some compact set $K \subset X$ ) and $r>0$ such that

$$
B(\bar{v}, r) \subset T_{B}(S ; x) \quad\left(\text { resp. }, \quad r B_{X} \subset T_{B}(S ; x)-K\right) \quad \forall x \in B(\bar{x}, r) \cap S .
$$

(b) If $X$ is Asplund, then the set $S$ is epi-Lipschitzian (resp., compactly epiLipschitzian) at $\bar{x}$ if and only if there exist some $\bar{v} \in X$ (resp., some compact set $K \subset X$ ) and $r>0$ such that

$$
B(\bar{v}, r) \subset T_{B}^{w}(S ; x) \quad\left(\text { resp., } r B_{X} \subset T_{B}^{w}(S ; x)-K\right) \quad \forall x \in B(\bar{x}, r) \cap S .
$$


5. The convex case. This section examines the $K$ directionally Lipschitzian property for convex functions and sets. It improves, in the complete space setting, Theorem 4.1 in [4]. Before stating the main result, let us recall that for a convex function $f: X \longrightarrow \mathbb{R} \cup\{+\infty\}$, which is finite at $x$, the directional derivative

$$
f^{\prime}(x ; v):=\lim _{t \rightarrow 0^{+}} t^{-1}[f(x+t v)-f(x)]
$$

exists in $\mathbb{R} \cup\{-\infty,+\infty\}$ for any $v \in X$ and that

$$
f^{\prime}(x ; v)=\inf _{t>0} t^{-1}[f(x+t v)-f(x)] .
$$

We start now with the following theorem. Some of its equivalences follow along the lines of [4, Theorem 4.1].

Theorem 5.1. Let $f: X \longrightarrow \mathbb{R} \cup\{+\infty\}$ be a proper lsc convex function, $\bar{x} \in$ $\operatorname{dom} f$, and let $K \subset X$ be a nonempty convex compact subset. Then the following are equivalent:

(a) The function $f$ is $K$ directionally Lipschitzian at $\bar{x}$.

(b) There exist $\beta \in \mathbb{R}, r>0$, and a neighborhood $U$ of $\bar{x}$ in $X$ such that $\inf _{v \in r K} f(x+v) \leq \beta$ for all $x \in U$.

(c) For some $r>0$ the function $x \mapsto \inf _{v \in r K} f(x+v)$ is finite and continuous at the point $\bar{x}$.

(d) For some $r>0$ the function $x \mapsto \inf _{v \in r K} f(x+v)$ is finite on some neighborhood $U$ of $\bar{x}$.

(e) For some $r>0$ the inclusion $0 \in \operatorname{int}(\operatorname{dom} f-r K-\bar{x})$ holds.

(f) There exist $\beta \in \mathbb{R}$ and $r>0$ such that

$$
\sup _{b \in r B_{X}} \inf _{v \in K} f^{\prime}(x ; b+v) \leq \beta \quad \forall x \in B(\bar{x}, r) \cap \operatorname{dom} f .
$$

(g) There exist $\beta \in \mathbb{R}$ and $r>0$ such that

$$
\sup _{b \in r B_{X}} \inf _{v \in K} d^{-} f(x ; b+v) \leq \beta \quad \forall x \in B(\bar{x}, r) \cap \operatorname{dom} f .
$$

Proof. Observe first that for any $r>0$ the function $x \rightarrow \varphi(x):=\inf _{v \in r K} f(x+v)$ is convex (because of the convexity of $f$ and $K$ ) and is also lsc. Indeed, fix any $x \in X$ and choose a sequence $\left(x_{n}\right)$ of $X$ converging to $x$ and such that $\liminf _{x^{\prime} \rightarrow x} \varphi\left(x^{\prime}\right)=$ $\lim _{n \rightarrow \infty} \varphi\left(x_{n}\right)$. By compactness of $K$ and lower semicontinuity of $f$, choose for each $n$ some $v_{n} \in K$ such that $\varphi\left(x_{n}\right)=f\left(x_{n}+v_{n}\right)$. By compactness of $K$ again, there exists some subsequence $\left(v_{s(n)}\right)$ that converges to some $v \in K$. According to the lower semicontinuity of $f$, we have

$$
f(x+v) \leq \liminf _{n \rightarrow \infty} f\left(x_{s(n)}+v_{s(n)}\right)=\lim _{n \rightarrow \infty} \varphi\left(x_{s(n)}\right)=\liminf _{x^{\prime} \rightarrow x} \varphi\left(x^{\prime}\right) .
$$

Since $v \in r K$, we obtain $\varphi(x) \leq f(x+v) \leq \liminf _{x^{\prime} \rightarrow x} \varphi\left(x^{\prime}\right)$, and hence $\varphi$ is lsc. Further, $K$ being compact, from Lemma 2.1 we can use $\inf _{K}$ instead of $\operatorname{linf}_{K}$.

(a) $\Rightarrow$ (b). Assume that (a) holds, and choose $\beta \in \mathbb{R}$ and $r>0$ such that

$$
\inf _{v \in K} t^{-1}[f(x+t b+t v)-f(x)] \leq \beta
$$

for all $\left.\left.x \in B_{f}(\bar{x}, r), t \in\right] 0, r\right]$, and $b \in r B_{X}$. Then taking $x=\bar{x}$ and $t=r$, we see that

$$
\inf _{v \in K} f(\bar{x}+r b+r v) \leq r \beta+f(\bar{x})
$$


that is, for $\beta^{\prime}:=r \beta+f(\bar{x})$

$$
\inf _{v \in r K} f(x+v) \leq \beta^{\prime} \quad \forall x \in B\left(\bar{x}, r^{2}\right) .
$$

The property (b) then holds with $\beta^{\prime}, r$, and $U=B\left(\bar{x}, r^{2}\right)$.

The function $\varphi$ being convex and with values in $\mathbb{R} \cup\{+\infty\}$, it is known that it is bounded from above on a neighborhood of $\bar{x}$ if and only if it is finite and continuous at $\bar{x}$. This says that $(\mathrm{b}) \Leftrightarrow(\mathrm{c})$.

The assertions (c), (d), and (e) are equivalent due to the convexity and the lsc property of the function $\varphi$ on the Banach space $X$ with $\operatorname{dom} \varphi=\operatorname{dom} f-r K$.

(b) $\Rightarrow$ (f). According to (b), let $\beta \in \mathbb{R}$ and $r, s>0$ such that $\inf _{v \in r K} f(x+v) \leq \beta$ for all $x \in B(\bar{x}, s)$. By lower semicontinuity of $f$, choose some positive number $r^{\prime}<\min \left\{\frac{s}{2}, \frac{s}{2 r}\right\}$ such that $f$ is bounded from below by some $\alpha \in \mathbb{R}$ on the ball $B\left(\bar{x}, r^{\prime}\right)$, and take any $x \in B\left(\bar{x}, r^{\prime}\right) \cap \operatorname{dom} f$. Then the inequality $f^{\prime}(x ; v) \leq f(x+v)-f(x)$ (see (5.1)) yields, for any $b \in r^{\prime} B_{X}$,

$$
\inf _{v \in K} f^{\prime}(x ; b+v)=r^{-1} \inf _{v \in r K} f^{\prime}(x ; r b+v) \leq r^{-1}\left(\inf _{v \in r K} f(x+r b+v)-f(x)\right) \leq \beta^{\prime}
$$

for $\beta^{\prime}:=r^{-1}(\beta-\alpha)$; that is, $(f)$ holds.

(f) $\Rightarrow(\mathrm{g})$. This implication is obvious since $d^{-} f(x ; v) \leq f^{\prime}(x ; v)$.

$(\mathrm{g}) \Rightarrow(\mathrm{a})$. This follows from Theorem 3.9 and the inclusion $B_{f}(\bar{x}, r) \subset \operatorname{dom} f \cap$ $B(\bar{x}, r)$. This completes the proof.

The following corollary characterizes some classes of $K$ directionally Lipschitzian convex functions.

Corollary 5.2. Let $f: X \longrightarrow \mathbb{R} \cup\{+\infty\}$ be a proper lsc convex function, and let $\mathcal{K}$ be a class of nonempty convex compact subsets of $X$ with $K+x \in \mathcal{K}$ and $\alpha K \in \mathcal{K}$ for all $K \in \mathcal{K}, x \in X$, and $\alpha \in] 0,+\infty[$ (i.e., $\mathcal{K}$ is stable under translation and positive scalar product). The following are equivalent:

(a) The function $f$ is $K$ directionally Lipschitzian at some $\bar{x} \in \operatorname{dom} f$ for some $K \in \mathcal{K}$.

(b) At any point $\bar{x} \in \operatorname{dom} f$ the function $f$ is $K$ directionally Lipschitzian for some $K \in \mathcal{K}$.

(c) For any (resp., some) point $\bar{x} \in \operatorname{dom} f$ there exist some $\beta \in \mathbb{R}, r>0$, and $K \in \mathcal{K}$ such that

$$
\inf _{v \in K} f(x+v) \leq \beta \quad \forall x \in B(\bar{x}, r) .
$$

(d) For some $K \in \mathcal{K}$ one has

$$
0 \in \operatorname{int}(\operatorname{dom} f-K) .
$$

(e) For any (resp., some) $\bar{x} \in \operatorname{dom} f$ there exist some $\beta \in \mathbb{R}, r>0$, and $K \in \mathcal{K}$ such that

$$
\sup _{b \in B_{X}} \inf _{v \in K} f^{\prime}(x ; b+v) \leq \beta \quad \forall x \in B(\bar{x}, r) \cap \operatorname{dom} f .
$$

(f) For any (resp., some) $\bar{x} \in \operatorname{dom} f$ there exist some $\beta \in \mathbb{R}, r>0$, and $K \in \mathcal{K}$ such that

$$
\sup _{b \in B_{X}} \inf _{v \in K} d^{-} f(x ; b+v) \leq \beta \quad \forall x \in B(\bar{x}, r) \cap \operatorname{dom} f .
$$


Proof. (b) $\Rightarrow$ (c). Assume (b) and choose by (b) in Theorem 5.1 some $\beta \in \mathbb{R}$, $r, s>0$ such that $\inf _{v \in r K} f(x+v) \leq \beta$ for all $x \in B(\bar{x}, s)$. Then (c) in the corollary holds with $r^{\prime}:=s$ and $K^{\prime}:=r K$.

(c) $\Rightarrow$ (d). When (c) in the corollary is satisfied we may apply (e) from Theorem 5.1 to get $0 \in \operatorname{int}(\operatorname{dom} f-K-\bar{x})$, which says that $(\mathrm{d})$ in the corollary holds with $K^{\prime}:=K-\bar{x}$.

Similar arguments yield (d) $\Rightarrow$ (b) and (d) $\Leftrightarrow$ (a). Finally, the equivalences between (e), (f), and (b) (resp., (a)) follow also directly from Theorem 5.1.

REMARK 5.3. We point out that the equivalence between (a), (b), and (c) in either Theorem 5.1 or Corollary 5.2 does not require the completeness of the space $X$.

As a direct consequence of Corollary 5.2, taking as set $K$ a singleton, we obtain the characterization of directionally Lipschitzian convex functions.

Corollary 5.4. Let $f: X \longrightarrow \mathbb{R} \cup\{+\infty\}$ be a lsc convex function. Then, the following statements are equivalent:

(a) $f$ is directionally Lipschitzian at some point of $\operatorname{dom} f$.

(b) $f$ is directionally Lipschitzian at any point of $\operatorname{dom} f$.

(c) The interior of $\operatorname{dom} f$ is nonempty.

(d) For any (resp., some) $\bar{x} \in \operatorname{dom} f$ there exist some $\bar{v} \in X, \beta \in \mathbb{R}$, and $r>0$ such that

$$
f^{\prime}(x ; v) \leq \beta \quad \forall x \in B(\bar{x}, r) \cap \operatorname{dom} f, v \in B(\bar{v}, r) .
$$

The above result recovers the characterization of the directionally Lipschitzian property given in [28, Proposition 3] for convex functions.

Taking now as set $K$ a compact set, Corollary 5.2 yields the following equivalences. They correspond to some characterizations of [4, Theorem 4.1].

Corollary 5.5. Let $f: X \longrightarrow \mathbb{R} \cup\{+\infty\}$ be a lsc convex function. Then, the following statements are equivalent:

(a) $f$ is compactly epi-Lipschitzian at some point of $\operatorname{dom} f$.

(b) $f$ is compactly epi-Lipschitzian at any point of $\operatorname{dom} f$.

(c) There exists a compact set $K \subset X$ such that

$$
0 \in \operatorname{int}(\operatorname{dom} f-K) \text {. }
$$

(d) For any (resp., some) $\bar{x} \in \operatorname{dom} f$ there exist some $\beta \in \mathbb{R}, r>0$, and some compact set $K$ of $X$ such that

$$
\sup _{b \in B_{X}} \inf _{v \in K} f^{\prime}(x ; b+v) \leq \beta \quad \forall x \in B(\bar{x}, r) \cap \operatorname{dom} f .
$$

Proof. The proof is direct from Corollary 5.2, the inequality $\inf _{\overline{c o} K} \leq \inf _{K}$, and the fact that $\overline{c o} K$ is compact whenever $K$ is compact.

For a class $\mathcal{K}$ of nonempty convex compact sets which is stable under translation and positive scalar product, the assertions of Corollary 5.2 yield the following equivalences for the $K$ directionally Lipschitzian behavior, with $K \in \mathcal{K}$, of convex sets.

Corollary 5.6. Let $S$ be a nonempty closed convex subset of $X$, and let $\mathcal{K}$ be a class of nonempty convex compact subsets of $X$ which is stable by translation and positive scalar product. Then the following are equivalent:

(a) The set $S$ is $K$ directionally Lipschitzian at some $\bar{x} \in S$ for some $K \in \mathcal{K}$.

(b) At any point $\bar{x} \in S$ the set $S$ is $K$ directionally Lipschitzian for some $K \in \mathcal{K}$.

(c) There exists some $K \in \mathcal{K}$ such that $0 \in \operatorname{int}(S-K)$. 
(d) There exists a finite set $K^{\prime}$ of points of some $K \in \mathcal{K}$ such that $0 \in \operatorname{int}(S-$ co $\left.K^{\prime}\right)$.

(e) For any (resp., some) point $\bar{x} \in S$ there exists $r>0$ such that

$$
r B_{X} \subset \operatorname{cl}(] 0,+\infty[(S-x))-K
$$

for some $K \in \mathcal{K}$ and all $x \in B(\bar{x}, r) \cap S$.

Proof. The equivalences between (a), (b), and (c) are direct consequences of Corollary 5.2, and the equivalence between (a) and (e) follows from Theorem 4.5. Finally, since $(d) \Rightarrow(c)$ is obvious, it remains to argue that $(c) \Rightarrow(d)$ is true. If (c) holds, we have some $r>0$ such that $2 r \bar{B}_{X} \subset S-K$. Choose a finite subset $K^{\prime} \subset K$ such that $K \subset K^{\prime}+r \bar{B}_{X}$. Then

$$
2 r \bar{B}_{X} \subset S-\operatorname{co} K^{\prime}+r \bar{B}_{X},
$$

and hence, according to the Rådström cancellation lemma [25], $r \bar{B}_{X} \subset S-\operatorname{co} K^{\prime}$. The proof is complete.

The characterizations of the epi-Lipschtizian property for convex sets are given in the following corollary through the choice of $\mathcal{K}$ in Corollary 5.6 as the class of all singletons in $X$. The equivalence between (a) and (c) recovers the characterization given in [28, Proposition 3].

Corollary 5.7. Let $S$ be a nonempty closed convex set. Then, the following assertions are equivalent:

(a) $S$ is epi-Lipschitzian at $\bar{x} \in S$.

(b) $S$ is epi-Lipschitzian at any point of $S$.

(c) The interior of $S$ is nonempty.

(d) For any (resp., some) point $\bar{x} \in S$ there exist $\bar{v} \in X$ and $r>0$ such that $B(\bar{v}, r) \subset \operatorname{cl}(] 0,+\infty[(S-x))$ for all $x \in B(\bar{x}, r) \cap S$.

Finally, the equivalences of the next corollary encompass in particular some earlier characterizations of the compactly epi-Lipschitzian property for convex sets in $[4$, Theorem 2.5].

Corollary 5.8. Let $S$ be a nonempty closed convex set. Then, the following assertions are equivalent:

(a) $S$ is compactly epi-Lipschitzian at $\bar{x} \in S$.

(b) $S$ is compactly epi-Lipschitzian at any point of $S$.

(c) There exists some compact set $K \subset X$ such that $0 \in \operatorname{int}(S-K)$.

(d) For any (resp., some) point $\bar{x} \in S$ there exists $r>0$ such that

$$
r B_{X} \subset \operatorname{cl}(] 0,+\infty[(S-x))-K
$$

for some compact set $K \subset X$ and all $x \in B(\bar{x}, r) \cap S$.

REMARK 5.9. Recalling that a closed set $S$ is locally convex at $\bar{x} \in S$ if there exists a neighborhood $U$ of $\bar{x}$ such that $S \cap U$ is convex, it is directly seen that, for this class of sets, the same characterizations in the above corollaries still hold with $S$ replaced by $S \cap U$.

6. $K$ directionally Lipschitzian property of optimal value function. In this section, we present an example where characterizations of Lipschitz-like properties, obtained in the previous sections, can be used in the analysis of the behavior of value functions in optimization problems. It is not the aim of this work to provide technical details in order to ensure the existence and to obtain the expressions 
of directional derivatives of value functions, a subject studied deeply in, among others, $[13,30,3]$ (and references therein). Our objective is to show how our approach can be used in this framework. The detailed study of more general mathematical programming and optimal control problems will be the subject of a future work.

Let $X$ be a Banach space, $U$ be a compact space, and $\phi: X \times U \longrightarrow \mathbb{R} \cup\{+\infty\}$ be a lsc function bounded from below. We will denote by $d_{x}^{-} \phi(x, y ; v)$ the lower Dini directional derivative of the function $\phi$ with respect to the first variable in the direction $v \in X$.

Consider the problem

$$
f(x):=\inf \{\phi(x, y): y \in U\}
$$

where $f: X \longrightarrow \mathbb{R}$ is the marginal value function of the minimization problem $(P(x))$. Let $U(x):=\{y \in U: f(x)=\phi(x, y)\}$. Observe that $U(x) \neq \emptyset$ for all $x \in X$.

Proposition 6.1. Let $\bar{x} \in \operatorname{dom} f$ be a point for which there exist $\beta \in \mathbb{R}$ and $r>0$ such that for each $x \in B_{f}(\bar{x}, r)$ there exist $y(x) \in U(x)$ and a bounded set $K_{x} \subset X$ satisfying

$$
\inf _{v \in K_{x}} d_{x}^{-} \phi(x, y(x) ; v+b) \leq \beta \quad \forall b \in r B_{X} .
$$

Then, if $K=\cup_{x \in B(\bar{x}, r)} K_{x}$ is a bounded set, one has that the value function $f$ is $\overline{\mathrm{co}} K$ directionally Lipschitzian at $\bar{x}$.

Proof. As in the proof of Theorem 5.1, the lsc property of $\phi$ and the compactness of $U$ imply that $f$ is lsc. For $x \in \operatorname{dom} f$, we have for any $t>0$ and $y \in U(x)$

$$
t^{-1}[f(x+t w)-f(x)] \leq t^{-1}[\phi(x+t w, y)-\phi(x, y)] \quad \forall w \in X,
$$

and hence, making $w \rightarrow v$ and $t \rightarrow 0^{+}$yields

$$
d^{-} f(x ; v) \leq d_{x}^{-} \phi(x, y ; v)
$$

which gives

$$
d^{-} f(x ; v) \leq \inf _{y \in U(x)} d_{x}^{-} \phi(x, y ; v) \quad \forall v \in X .
$$

Now take $\beta \in \mathbb{R}$ and $r>0$ from the hypothesis and fix any $x \in B_{f}(\bar{x}, r)$. Taking $y(x) \in U(x)$ satisfying assumption (6.1) of the proposition, we have from (6.2) that

$$
\inf _{v \in K_{x}} d^{-} f(x ; v+b) \leq \inf _{v \in K_{x}} d_{x}^{-} \phi(x, y(x) ; v+b) \leq \beta \quad \forall b \in r B_{X},
$$

and hence

$$
\inf _{v \in K} d^{-} f(x ; v+b) \leq \beta \quad \forall b \in r B_{X}, \forall x \in B_{f}(\bar{x}, r) .
$$

The above inequality and the boundedness of $K$ allow us to conclude, according to Proposition 3.6, that $f$ is $\overline{\mathrm{co}} K$ directionally Lipschitzian at $\bar{x}$.

7. Conclusions. In this work we have obtained, in a unified way, equivalences for various Lipschitz-like properties, following an approach that also provides several new characterizations as well as some new Lipschitz-like properties. In order to accomplish this, we introduced a general notion that we call the $K$ directionally 
Lipschitzian property which covers, for functions, the concepts of locally Lipschitzian, directionally Lipschitzian, and compactly epi-Lipschitzian properties, and for sets, the epi-Lipschitzian and compactly epi-Lipschitzian concepts. The characterizations of the $K$ directionally Lipschitzian property for a function are related to some mixed boundedness property of its lower Dini directional derivative, and for a set they are related to the interior of the Minkowski sum of its Bouligand tangent cone and some compact set.

For characterizations of $K$ Lipschitz-like properties of functions in terms of subdifferentials which are not (necessarily) generated by generalized directional derivatives as support functions, we refer to paper [10] which is a continuation of this one.

\section{REFERENCES}

[1] D. Aussel, J.-N. Convellec, And M. Lassonde, Nonsmooth constrained optimization and multidirectional mean value inequalities, SIAM J. Optim., 9 (1999), pp. 690-706.

[2] E. Bishop And R. R. Phelps, The support functionals of a convex set, in Proc. Sympos. Pure Math., Vol. VII, Amer. Math. Soc., Providence, RI, 1963, pp. 27-35.

[3] J. F. Bonnans and A. Shapiro, Perturbation Analysis of Optimization Problems, Springer Ser. Oper. Res., Springer-Verlag, New York, 2000.

[4] J. Borwein, Y. LuCet, AND B. Mordukhovich, Compactly epi-Lipschitzian convex sets and functions in normed spaces, J. Convex Anal., 7 (2000), pp. 375-393.

[5] J. M. Borwein And A. S. Lewis, Partially finite convex programming. I. Quasi relative interiors and duality theory, Math. Programming, 57 (1992), pp. 15-48.

[6] J. M. Borwein And H. M. Stróswas, Tangential approximations, Nonlinear Anal., 9 (1985), pp. $1347-1366$.

[7] J. M. Borwein And H. M. Strójwas, Proximal analysis and boundaries of closed sets in Banach space. II. Applications, Canad. J. Math., 39 (1987), pp. 428-472.

[8] F. H. Clarke and Yu. S. Ledyaev, Mean value inequalities in Hilbert space, Trans. Amer. Math. Soc., 344 (1994), pp. 307-324.

[9] B. Connet, Regularity Properties of Normal and Tangent Cones, thesis, Université de ParisDauphine, Paris, 1981.

[10] R. Correa, P. Gajardo, and L. Thibault, Various Lipschitz like properties for functions and sets II: Subdifferential and normal characterizations, to appear.

[11] R. Correa, P. Gajardo, And L. Thibault, Subdifferential representation formula and subdifferential criteria for the behavior of nonsmooth functions, Nonlinear Anal., 65 (2006), pp. 864-891.

[12] R. Correa, P. Gajardo, and L. Thibault, Links between directional derivatives through multidirectional mean value inequalities, Math. Program., Ser. B, 116 (2009), pp. 57-77.

[13] R. Correa And A. Seeger, Directional derivative of a minimax function, Nonlinear Anal., 9 (1985), pp. 13-22.

[14] N. Dunford And J. T. Schwartz, Linear operators. Part I. General theory. With the assistance of William G. Bade and Robert G. Bartle, reprint of the 1958 original, Wiley Classics Library, a Wiley-Interscience Publication, John Wiley \& Sons, New York, 1988.

[15] A. D. Ioffe, Approximate subdifferentials and applications. III. The metric theory, Mathematika, 36 (1989), pp. 1-38.

[16] A. D. Ioffe, Codirectional compactness, metric regularity and subdifferential calculus, in Constructive, Experimental, and Nonlinear Analysis (Limoges, 1999), CMS Conf. Proc. 27, Amer. Math. Soc., Providence, RI, 2000, pp. 123-163.

[17] A. D. Ioffe, Metric regularity and subdifferential calculus, Uspekhi Mat. Nauk, 55 (2000), pp. 103-162.

[18] A. Jourani and L. Thibault, Metric regularity for strongly compactly Lipschitzian mappings, Nonlinear Anal., 24 (1995), pp. 229-240.

[19] A. Jourani And L. Thibault, Extensions of subdifferential calculus rules in Banach spaces, Canad. J. Math., 48 (1996), pp. 834-848.

[20] P. D. Loewen, The proximal subgradient formula in Banach space, Canad. Math. Bull., 31 (1988), pp. 353-361.

[21] P. D. Loewen, Limits of Fréchet normals in nonsmooth analysis, in Optimization and Nonlinear Analysis, Pitman Res. Notes Math. 244, Longman Scientific and Technical, Harlow, UK, 1992, pp. 178-188. 
[22] B. S. Mordukhovich, Variational Analysis and Generalized Differentiation. I. Basic Theory, Grundlehren Math. Wiss. 330, Springer-Verlag, Berlin, 2006.

[23] B. S. Mordukhovich And Y. ShaO, Stability of set-valued mappings in infinite dimensions: Point criteria and applications, SIAM J. Control Optim., 35 (1997), pp. 285-314.

[24] J.-P. Рenot, A characterization of tangential regularity, Nonlinear Anal., 5 (1981), pp. 625643.

[25] H. RÅdström, An embedding theorem for spaces of convex sets, Proc. Amer. Math. Soc., 3 (1952), pp. 165-169.

[26] R. T. Rockafellar, Clarke's tangent cones and the boundaries of closed sets in $\mathbf{R}^{n}$, Nonlinear Anal., 3 (1978), pp. 145-154.

[27] R. T. Rockafellar, Directionally Lipschitzian functions and subdifferential calculus, Proc. London Math. Soc. (3), 39 (1979), pp. 331-355.

[28] R. T. Rockafellar, Generalized directional derivatives and subgradients of nonconvex functions, Canad. J. Math., 32 (1980), pp. 257-280.

[29] R. T. Rockafellar, Proximal subgradients, marginal values, and augmented Lagrangians in nonconvex optimization, Math. Oper. Res., 6 (1981), pp. 424-436.

[30] R. T. Rockafellar, Directional differentiability of the optimal value function in a nonlinear programming problem: Sensitivity and parametric analysis, Math. Programming Stud., 21 (1984), pp. 213-226.

[31] R. T. Rockafellar and R. J.-B. Wets, Variational Analysis, Grundlehren Math. Wiss. 317, Springer-Verlag, Berlin, 1998.

[32] L. Thibault, A note on the Zagrodny mean value theorem, Optimization, 35 (1995), pp. 127130.

[33] L. Thibault and D. Zagrodny, Integration of subdifferentials of lower semicontinuous functions on Banach spaces, J. Math. Anal. Appl., 189 (1995), pp. 33-58.

[34] L. Thibault and N. Zlateva, Integrability of subdifferentials of directionally Lipschitz functions, Proc. Amer. Math. Soc., 133 (2005), pp. 2939-2948.

[35] J. S. Treiman, Characterization of Clarke's tangent and normal cones in finite and infinite dimensions, Nonlinear Anal., 7 (1983), pp. 771-783.

[36] J. S. Treiman, Generalized gradients, Lipschitz behavior and directional derivatives, Canad. J. Math., 37 (1985), pp. 1074-1084.

[37] D. Zagrodny, Approximate mean value theorem for upper subderivatives, Nonlinear Anal., 12 (1988), pp. 1413-1428.

[38] Q. ZHU, Clarke-Ledyaev mean value inequalities in smooth Banach spaces, Nonlinear Anal., 32 (1998), pp. 315-324. 